\title{
Obstrucción intestinal crónica por banda congénita en paciente preescolar
}

\author{
Intestinal chronic obstruction by congenital band in a preschool patient
}

\author{
Katherine Márquez-Bayona,*,+ Katherine Sofía Anaya-Pinzón,* Luis Augusto Zárate-Suárez* \\ * Hospital Universitario de Santander, Bucaramanga, Santander, Colombia.
}

\begin{abstract}
RESUMEN
Introducción: En la edad pediátrica, las bandas congénitas anómalas (BCA) presentan diferentes manifestaciones abdominales. Su diagnóstico y tratamiento son eminentemente quirúrgicos. Caso clínico: Paciente de cinco años con varios episodios de obstrucción intestinal desde los 16 meses de edad, que se resolvieron con manejo conservador, aun cuando se requirió hospitalización. Se sospechó de enfermedad de Hirschsprung, pero no se pudo realizar estudios para confirmar diagnóstico. Por cuadro de abdomen agudo fue llevado a cirugía, teniendo como hallazgo una BCA, la cual ocasionaba hernia interna y dilatación de asas intestinales. Se procedió a su resección y anastomosis término-lateral. Posteriormente el paciente tuvo una adecuada evolución clínica. Conclusiones: El diagnóstico de BCA es una condición que puede ser de difícil diagnóstico, dado lo inespecífico del cuadro clínico y porque los estudios de imagen resultan de poca utilidad. De esta forma, debería ser parte del diagnóstico diferencial en pacientes con datos de oclusión intestinal.
\end{abstract}

Palabras clave: Obstrucción intestinal, banda congénita anómala, dolor abdominal, preescolar.

\section{INTRODUCCIÓN}

Las bandas congénitas anómalas (BCA) se consideran una causa extremadamente rara de obstrucción intestinal aguda, ${ }^{1}$ siendo las adherencias postoperatorias

\footnotetext{
+ Correspondencia: KMB, kathemqz@gmail.com

Conflicto de intereses: Los autores declaran que no tienen. Citar como: Márquez-Bayona K, Anaya-Pinzón KS, Zárate-Suárez LA. Obstrucción intestinal crónica por banda congénita en paciente preescolar. Rev Mex Pediatr. 2020; 87(4): 146-149. https:// dx.doi.org/10.35366/95825
}

\begin{abstract}
Introduction: Congenital abnormal bands (CAB) have different abdominal manifestations in children. Diagnosis and treatment are surgical. Clinical case: A five-year-old patient with history of episodes of intestinal obstruction from 16 months of age, which were resolved by conservative management, despite requiring hospitalization. Hirschsprung's disease was suspected, but studies were not performed to confirm the diagnosis. For acute abdomen was taken to surgery; during the procedure we found a $C A B$, which caused internal hernia and dilated intestinal loops. We proceeded to perform the $C A B$ resection and end-to-side anastomosis. Subsequently, the patient had adequate clinical evolution. Conclusions: The diagnosis of CAB can be difficult, given the nonspecific clinical picture and because imaging studies are not useful. Thus, CAB should be part of the differential diagnosis in children with evidence of intestinal obstruction.
\end{abstract}

Keywords: Intestinal obstruction, congenital anomaly band, abdominal pain, children.

las que aportan $80 \%$ de los casos de obstrucción a nivel del intestino delgado. ${ }^{2}$ Sin embargo, en la obstrucción intestinal no solo existe un cuadro clínico agudo, ya que es posible la presencia de vómitos cíclicos de características biliosas, dolor abdominal crónico, retraso en el crecimiento, constipación intestinal y datos de abdomen agudo. ${ }^{3-5}$

$\mathrm{Su}$ etiología hasta el momento es desconocida; ${ }^{6}$ algunos autores consideran que son remanentes de vasos fetales y del mesenterio central que no fueron reabsorbidos normalmente, ${ }^{7}$ aunque no todos coinciden. ${ }^{6}$ En lo que sí hay consenso es que se produce el 


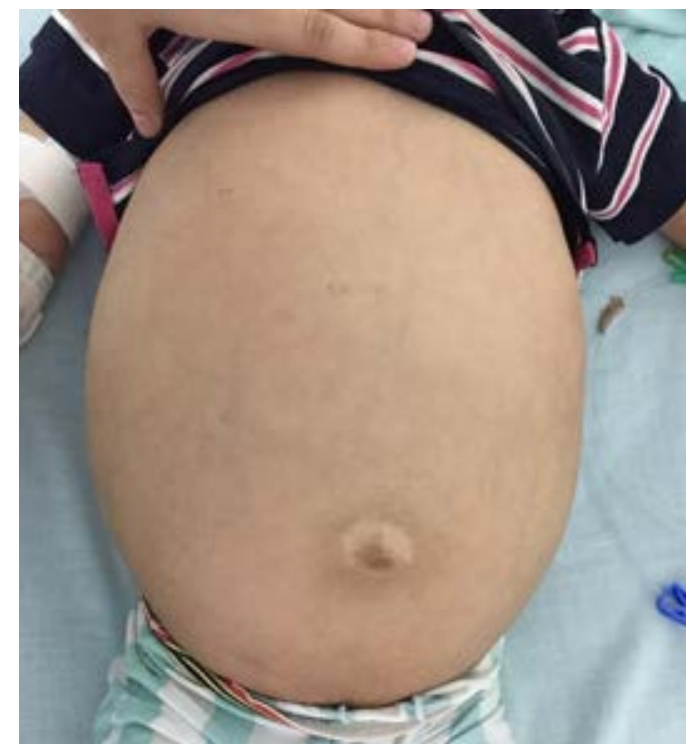

Figura 1: Marcada distensión abdominal al examen físico.

atrapamiento de un asa intestinal entre la banda y el mesenterio. ${ }^{2}$ La localización más frecuente es a nivel del íleon terminal, con casos reportados en colon ascendente, colon sigmoides y yeyuno proximal. ${ }^{4}$

Teniendo en cuenta el espectro de manifestaciones, se encuentra una amplia variedad de diagnósticos diferenciales como malformaciones anorrectales, invaginación intestinal, divertículo de Meckel, tuberculosis intestinal, enfermedad de Hirschsprung, síndrome de malrotación intestinal y ascariasis, según la edad del paciente. $^{8}$

El diagnóstico y tratamiento son quirúrgicos; los estudios de imagen son poco útiles para el diagnóstico y pueden producir retraso en el manejo definitivo, que consiste en la resección del segmento comprometido. ${ }^{2}$

A continuación, se presenta un caso de obstrucción intestinal crónica por BCA a nivel de íleon, señalando cómo se llegó al diagnóstico, y que los hallazgos intraoperatorios justificaron la realización de resección intestinal y anastomosis término-terminal.

\section{PRESENTACIÓN DEL CASO}

Paciente masculino de cinco años de edad, con antecedente de dos hospitalizaciones previas a la edad de 16 meses y tres años por cuadros de obstrucción intestinal, sospechando enfermedad de Hirschsprung. Sin embargo, este diagnóstico no se llegó a establecer por histopatología por dificultades administrativas y familiares que impidieron la toma de biopsias por cirugía, así como pérdida del seguimiento por lejanía del sitio de vivienda.

El cuadro actual fue de un mes de evolución con astenia, adinamia e hiporexia. Por sospecha de síndrome linfoproliferativo fue remitido a urgencias pediátricas de un hospital de tercer nivel.

Como parte del interrogatorio, se documentan cuadros intermitentes de ausencia de evacuaciones, distensión abdominal marcada y desnutrición proteicocalórica (Figura 1).

Se realiza radiografía de abdomen en la que se observa distensión de asas intestinales y ausencia de gas distal (Figura 2). Por lo anterior, el Servicio de Cirugía Pediátrica considera que, dados los antecedentes y estado del paciente, se requiere de un procedimiento quirúrgico, a fin de establecer el diagnóstico y, probablemente, resolver el problema.

Se realizó laparotomía exploratoria, observando dilatación importante de todo el intestino delgado, hasta $50 \mathrm{~cm}$ de la válvula ileocecal, debido a un segmento con brida que producía una hernia interna (Figura 3A), la cual ocasionaba dilatación de aproximadamente $30 \times$ $10 \times 10 \mathrm{~cm}$ (Figura 3B), sin permitir un adecuado paso de gas ni líquido. Por esos hallazgos se decidió resección de bolsa ileal a $20 \mathrm{~cm}$ de la válvula ileocecal, efectuando

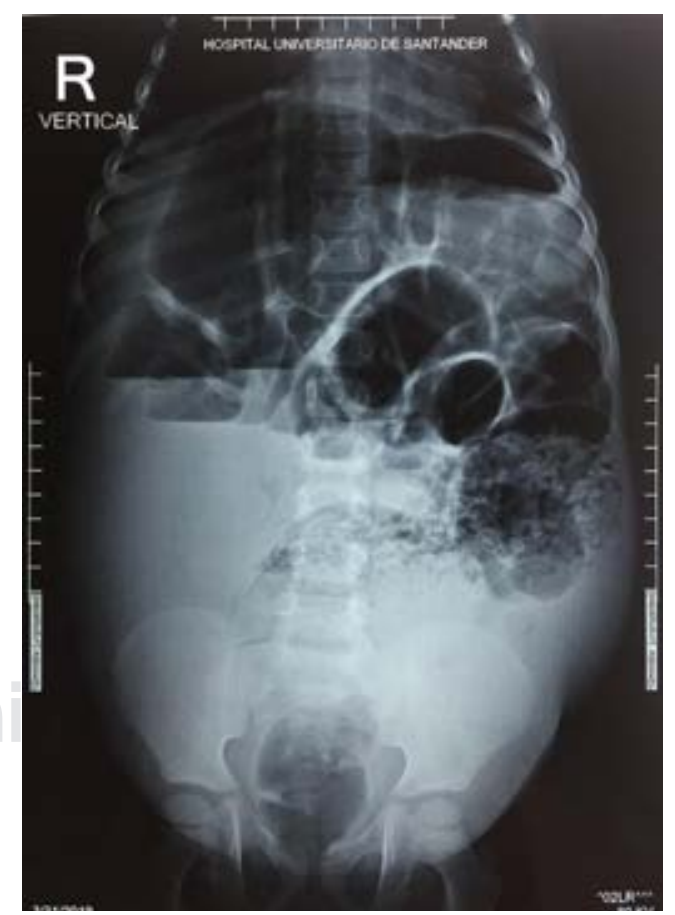

Figura 2: Radiografía de abdomen en la que se observa distensión de asas con contenido intestinal y ausencia de gas distal. 

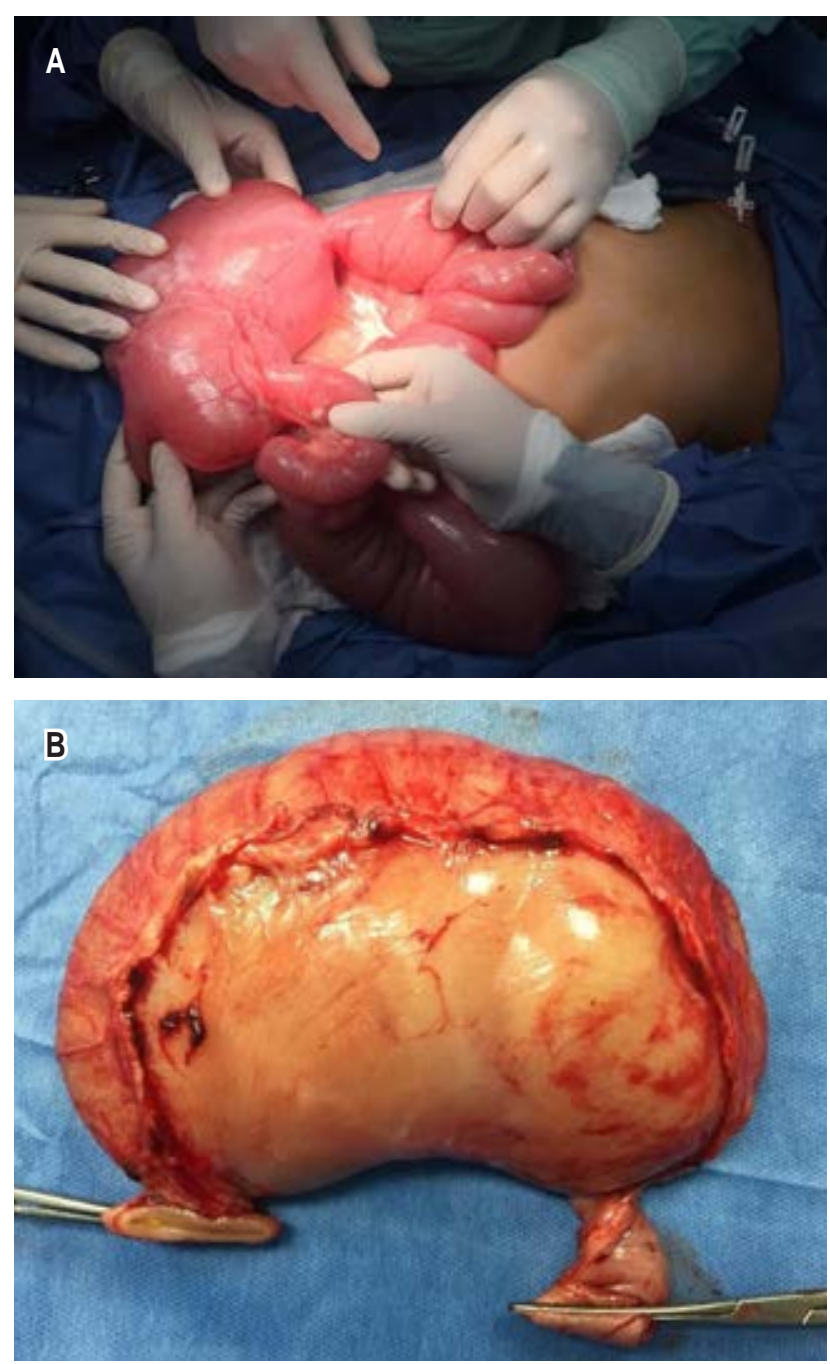

Figura 3: Hallazgos intraoperatorios. A) Se observa el segmento de intestino delgado marcadamente disminuido de calibre por presencia de bridas. B) Segmento resecado.

anastomosis término-lateral. Antes de terminar, se verificó el tránsito intestinal en íleon distal y colon, aunque con cierta dificultad.

El paciente tuvo buena evolución postoperatoria, con marcada mejoría de distensión abdominal, así como en la frecuencia y características de las evacuaciones.

\section{DISCUSIÓN}

El diagnóstico de BCA constituye un reto diagnóstico. Representa una de las múltiples causas de obstrucción intestinal identificadas en población pediátrica; ${ }^{9} \sin$ embargo, las manifestaciones clínicas varían desde una presentación sintomática leve hasta la estrangulación del intestino. Se presenta a cualquier edad, desde la etapa neonatal, siendo extremadamente rara en adultos. Las manifestaciones son más tempranas cuando hay complicaciones, como vólvulos o estrangulación. ${ }^{2,3}$

Existen pocas series de casos que describen las características de las BCA y se cuenta con muy pocos casos reportados en la literatura reciente. En la primera serie, se describen ocho pacientes (siete masculinos y uno femenino) de seis días a seis años de edad; todos mostraban signos y síntomas sugestivos de obstrucción intestinal, mientras que los pacientes mayores de dos años de edad tenían historia clínica de dolor abdominal crónico. ${ }^{6}$ Erginel y col. describen 14 pacientes (10 masculinos y cuatro femeninos) que ingresaron con diagnóstico clínico de obstrucción intestinal aguda, con edades entre los cuatro días y los 12 años (incluyendo dos neonatos), y que presentaban distensión abdominal, dolor abdominal y vómito. ${ }^{7} \mathrm{En}$ el caso que nos ocupa, el paciente tenía una historia de larga evolución de obstrucción intestinal y con constipación, lo cual era intermitente por lo que se le prestaba poca importancia por parte de la familia. En las ocasiones que llegó a hospitalizarse, los padres no aceptaban la realización de un procedimiento quirúrgico para descartar enfermedad de Hirschsprung, lo que dificultó al equipo médico brindarle la mejor atención posible.

Hay estudios donde los hallazgos histopatológicos demuestran que las bandas están compuestas por tejido altamente vascularizado, sugiriendo su origen congénito. ${ }^{3}$ Dell y col. describieron cuatro tipos de bandas congénitas peritoneales, basadas en su localización y compromiso a nivel del intestino delgado. La banda tipo 1, también conocida como "banda de Ladd", en la cual el ciego se encuentra anormalmente ubicado en el cuadrante superior derecho del abdomen. La banda se extiende a través de la segunda y la tercera parte del duodeno hasta el canal paravertebral. Por su parte, la banda tipo 2 se caracteriza por extenderse desde la flexura hepática del colon a través de la segunda parte del duodeno, hasta el canal paravertebral derecho; mientras que en la tipo 3 se describe como hipertrofia de los ligamentos hepatoduodenales. Finalmente, la banda tipo 4 se describe como bandas fibrosas densas que unen la porción distal de la tercera parte del duodeno a la fascia paravertebral. ${ }^{9}$

Diversos autores están de acuerdo en que el sitio más frecuente de estas bandas se encuentra entre el colon ascendente y el íleon terminal, seguido de la localización en el ligamento de Treitz y el mesenterio 
del íleon terminal. En menor frecuencia (hasta 25\%) la banda se origina en el hígado y se adhiere al mesenterio. ${ }^{6-10}$ El paciente del caso expuesto presentaba dichas bandas comprometiendo el íleon terminal, lo que concuerda con la literatura en cuanto a la localización principal.

Es importante tener en cuenta que la obstrucción intestinal se produce por tres mecanismos fundamentales: compresión del intestino (más de 50\% de los casos), vólvulo parcial (alrededor de 30\%) y atrapamiento del asa intestinal entre la banda y el mesenterio, estos últimos en alrededor de $30 \%$ de los casos. ${ }^{6-11}$ En el caso presentado, el mecanismo que ocasionó los periodos de obstrucción intestinal fue el del vólvulo parcial, que se observó claramente en los hallazgos quirúrgicos.

Se ha documentado que los estudios de imagen no son útiles para el diagnóstico. ${ }^{2}$ Sin embargo, la tomografía se utiliza para excluir otras causas de obstrucción, ${ }^{4}$ pero la exploración quirúrgica es el estándar para el diagnóstico y tratamiento.

En cuanto al tratamiento, en algunos casos la escisión quirúrgica de la brida suele ser suficiente, mientras que cuando se presenta necrosis intestinal secundaria a la compresión de la banda, se requiere resección intestinal. ${ }^{6}$ La cirugía puede ser por laparotomía o laparoscopía. ${ }^{2-14}$ La mortalidad asociada a BCA puede llegar al 10\%, y está directamente relacionada con un retraso en el diagnóstico y tratamiento definitivo. $^{2}$

\section{REFERENCIAS}

1. Galván-Montaño A, Trejo-Ávila M, García-Moreno S, Pérez A. Banda congénita anómala una patología rara de obstrucción intestinal en niños. Caso clínico. Cir Ciruj. 2017; 85(2): 164-167. http://dx.doi.org/10.1016/j.circir.2015.10.011.

2. Yang KH, Lee TB, Lee SH, Kim SH, Cho YH, Kim HY. Congenital adhesion band causing small bowel obstruction: What's the difference in various age groups, pediatric and adult patients? BMC Surg. 2016;16(1):79.
3. Kostic A, Krstic M, Slavkovic A, Vacic N. Intestinal obstruction in children could it be congenital abdominal bands? Pediatr Emerg Care. 2013; 29: 500-501. doi: 10.1097/PEC.0b013e31828a388f.

4. Chang YT, Chen BH, Shih HH, Hsin YM, Chiou CS. Laparoscopy in children with acute intestinal obstruction by aberrant congenital bands. Surg Laparosc Endosc Percutan Tech. 2010; 20(1): e34-e37. doi: 10.1097/SLE.0b013e3181cdb89a.

5. Dassinger MS, Smith SD. Disorders of intestinal rotation and fixation. In: Coran AG, Grosfeld JL, O'Neill JA Jr, Fonkalsrud EW, eds. Pediatric Surgery, 7th ed. Philadelphia: Mosby, 2012; 1111-1125. doi: 10.1016/B978-0-323-07255-7.00086-6.

6. Erginel B, Soysal FG, Ozbey H, Keskin H, Celik A, Karadag A, et al. Small bowel obstruction due to anomalous congenital bands in children. Gastroenterol Res Pract. 2016; 2016:7364329. doi: 10.1155/2016/7364329.

7. Aydin E. A rare cause of intestinal obstruction in a newborn: congenital band compression. North Clin Istanbul. 2016; 3 (1): 75-78. doi: 10.14744/nci.2015.26349.

8. Ghritlaharey RK, Budhwani KS, Shrivastava DK. Exploratory laparotomy for acute intestinal conditions in children: A review of 10 years of experience with 334 cases. Afr J Paediatr Surg. 2011; 8: 62-69. https://doi.org/10.4103/0189-6725.78671.

9. Maiese A, Bonaccorso L, Dell'Aquila M, Gitto L, Bolino G. Anomalous congenital band and intestinal obstruction: report of a fatal case in a child. Forensic Sci Med Pathol. 2013;9(4):588-590. doi:10.1007/s12024-013-9461-3.

10. Akgür FM, Tanyel FC, Büyükpamukçu N, Hiçsönmez A. Anomalous congenital bands causing intestinal obstruction in children. J Pediatr Surg. 1992;27 (4):471-473. doi:10.1016/00223468(92)90340-d

11. Nicolas G, Kfoury T, Shimlati R, Koury E, Tohme M, Gharios E, et al. Diagnosis and treatment of small bowel strangulation due to congenital band: three cases of congenital band in adults lacking a history of trauma or surgery. Am J Case Rep. 2016; 17: 712-719. doi: 10.12659/AJCR.899664.

12. Etensel B, Özkisacik S, Döger F, Yazici M, Gürsoy H. Anomalous congenital band: a rare cause of intestinal obstruction and failure to thrive. Pediatr Surg Int. 2005; 21: 1018-1020. doi: 10.1007/ s00383-005-1563-x.

13. Fang AC, Carnell J, Stein JC. Constipation in a 7-year-old boy: Congenital band causing a strangulated small bowel and pulseless electrical activity. J Emerg Med. 2012; 42(3): 283-287. doi: 10.1016/j.jemermed.2010.05.092.

14. Jerraya $H$, Khalfallah M, Gaja A, Dziri C. Laparoscopic treatment of intestinal obstruction caused by an uncommon congenital band. BMJ Case Rep. 2015; 2015: bcr2015212536. doi: 10.1136/bcr2015-212536. 